\title{
SUBRINGS OF NOETHERIAN RINGS
}

\section{EDWARD FORMANEK AND ARUN VINAYAK JATEGAONKAR}

ABSTRACT. Let $S$ be a subring of a ring $R$ such that $R$ is a finitely generated right $S$-module. Clearly, if $S$ is a right Noetherian ring then so is $R$. Generalizing a result of P. M. Eakin, we show that if $R$ is right Noetherian and $S$ is commutative then $S$ is Noetherian. We also show that if $R_{S}$ has a finite generating set $\left\{u_{1}, \ldots, u_{m}\right\}$ such that $u_{i} S=$ $S u_{i}$ for $1 \leq i \leq m$, then a right $R$-module is Noetherian, Artinian or semisimple iff it is respectively so as a right $S$-module. This yields a result of Clifford on group algebras.

Let $S$ be a subring of a ring $R$ such that $R$ is finitely generated as a right $S$-module. It is well known (and trivial) that if $S$ is a right Noetherian ring then $R$ is a right Noetherian ring. The converse is false in general as can be seen by taking $R=\left(\begin{array}{ll}\mathbf{Q} & \mathbf{Q} \\ \mathbf{Q} & \mathbf{Q}\end{array}\right)$ and $S=\left(\begin{array}{cc}\mathbf{Q} & \mathbf{Q} \\ 0 & \mathrm{Z}\end{array}\right)$. However, P. M. Eakin [3] (and later M. Nagata [10]) showed that the converse holds if $R$ is assumed to be a commutative ring. D. Eisenbud [4] and J. E. Björk [1] have extended Eakin's theorem to some mildly noncommutative situations.

In this note, we provide two mildly noncommutative versions of Eakin's theorem. The first version answers a question raised by J. E. Björk [8]. The second version improves upon Eisenbud's generalization of Eakin's theorem and, when applied to group algebras, yields a theorem of Clifford [2, p. 343].

As usual, all rings, subrings and modules are assumed to be unitary. Recall that a module $M_{R}$ is finite dimensional if it does not contain any infinite direct sum of nonzero submodules. It is known $[7, \mathrm{p} .216]$ that if $M_{R}$ is finite dimensional then there exists a nonnegative integer $n$ such that any direct sum of nonzero submodules of $M$ contains at most $n$ terms. The least such integer is called the uniform dimension of $M$ and is denoted as $d(M)$ or $d_{R}(M)$.

Received by the editors September 17, 1973.

AMS(MOS) subject classifications (1970). Primary 16A46, 13E05; Secondary 16 A26, 16 A38.

Key words and phrases. Commutative Noetherian rings, noncommutative Noetherian rings, P. I. rings, Eakin's theorem, Clifford's theorem on group algebras. 
Our proofs of our main results have a certain strategy in common. In each case, we start with a Noetherian object and want to show that some related object is Noetherian. Due to the Noetherian induction, this presents no problem in lots of situations. Once the dubious cases are singled out, we show that the following lemma or the idea underlying its proof is applicable.

1. Lemma. Let $M$ be a finite dimensional module. If $M / N$ is Noetherian for every essential submodule $N$ of $M$ then $M$ is Noetherian.

Proof. Let $\left\{M_{n}: n \geq 1\right\}$ be an ascending chain of submodules of $M$. Since $d\left(M_{n}\right)$ is a nondecreasing sequence of nonnegative integers which is bounded above by $d(M)$, it is eventually constant; say, $d\left(M_{n}\right)=d\left(M_{m}\right)$ for all $n \geq m$. We can use the finite dimensionality of $M$ (or Zorn's lemma) to obtain a submodule $K$ of $M$ such that the sum $K+M_{m}$ is direct and essential in $M$. For $n \geq m$, we have $\left(K \cap M_{n}\right) \oplus M_{m} \subseteq M_{n}$ and $d\left(M_{n}\right)=d\left(M_{m}\right)$. It follows that $K \cap M_{n}=(0)$. Since the chain $\left\{K \oplus M_{n}: n \geq 1\right\}$ stops, so does $\left\{M_{n}: n \geq 1\right\}$.

The following lemma is essentially Eakin's theorem stated for modules rather than rings. Its proof illustrates the strategy indicated above.

2. Lemma. Let $S$ be a subring of a commutative Noetherian ring $R$. Then any $R$-module which is finitely generated over $S$ is Noetherian over $S$.

Proof. Let $M$ be an $R$-module which is finitely generated over $S$. Assume that every proper $R$-homomorphic image of $M$ is Noetherian over $S$. We proceed to show that $M$ itself is Noetherian over $S$.

Among the nonzero $R$-submodules of $M$ which are finitely generated over $S$, choose $L$ with largest possible $a_{n} n_{R}$. Since $M / L$ is Noetherian over $S$, it suffices to show that $L$ is Noetherian over $T=S / \operatorname{ann}_{S} L$. Note that $T$ is a domain. For, if $x, y$ are nonzero elements of $T$ such that $x y=0$ then $L x$ is a nonzero $R$-submodule of $M$ which is finitely generated over $S$ and annihilated by $y$, contradicting our choice of $L$.

Consider the $T$-torsion submodule $N$ of $L$. It is in fact an $R$-submodule of $L$. Further, it is a proper submodule since $L$ is finitely generated and faithful over the domain $T$. If $N \neq(0)$ then $L / N$ is a (torsionfree and so) faithful Noetherian $T$-module. It follows that $T$ is a Noetherian ring $[9$, p. 53], and so $L$ is Noetherian over $T$.

It remains to treat the case when $N=(0)$. In this case, $L$ is finitely generated and torsion-free over $T$; so, it is finite dimensional over $T$. If 
$K$ is any essential $T$-submodule of $L$ then $L / K$ is finitely generated and torsion so unfaithful over $T$. This yields a nonzero $t \in T$ such that $L t \subseteq$ $K$. Since $L t$ is a $R$-submodule of $L, L / L t$ and so $L / K$ is Noetherian over. $T$. Lemma 1 shows that $L$ is Noetherian over $T$. Now a routine Noetherian induction proves the lemma.

An alternate proof of Lemma 2 can be obtained by using the main result of [5]. We now prove a version of Eakin's theorem which answers a question of J. E. Björk [8, p. 376].

3. Theorem. Let $S$ be a commutative subring of a right Noetherian ring $R$ such that $R$ is finitely generated as a right $S$-module. Then $S$ is a Noetherian ring.

Proof. Clearly, it suffices to show that $R$ is Noetherian as a right $S$-module. In view of the Noetherian induction, we may assume without loss that for every two-sided ideal $I$ of $R, R / I$ is Noetherian as a right $S$-module.

Suppose $R$ is not a prime ring. Then there exists a finite number of prime ideals $P_{1}, \ldots, P_{k}$ of $R$ such that $\bigcap\left\{P_{i}: 1 \leq i \leq k\right\}$ is the prime radical $P(R)$ of $R$. By Levitski's theorem, $\{P(R)\}^{\bar{l}}=(0)$ for some positive integer $l$. It is clear that $\{P(R)\}^{i-1} /\{P(R)\}^{i}, 1 \leq i \leq l$, is an $R$-homomorphic image of a finite direct sum of the right $R$-module $R / P(R)$. Thus, if $P(R) \neq(0)$ then $R_{S}$ is Noetherian. If $P(R)=(0)$ then we must have $k>1$ since $R$ is not a prime ring. Then $R$ embeds in $\Pi\left\{R / P_{i}: 1 \leq i \leq k\right\}$ as a right $R$-module which makes $R_{S}$ Noetherian.

We now treat the (dubious) case when $R$ is a prime ring. A result due to Procesi and Small [11] shows that End $R_{S}$ satisfies a polynomial identity. Since $R$ is isomorphic with a subring of End $R_{S}, R$ is a prime P.I. ring. A theorem due to Formanek [6] now shows that there exists a $Z(R)$ monomorphism $R \hookrightarrow Z(R)^{(m)}$ for some positive integer $m$. Since the centre $Z(R)$ of $R$ is a domain, it follows that $R$ is a finite dimensional $Z(R)$ module.

Set $T=S Z(R)$. Let $\left\{M_{n}: n \geq 1\right\}$ be an ascending chain of $T$-submodules of $R$. There exists a positive integer $h$ such that $d_{Z(R)}\left(M_{n}\right)=$ $d_{Z(R)}\left(M_{b}\right)$ for all $n \geq h$. Choose a $Z(R)$-submodule $K$ of $R$ such that the sum $K+M_{b}$ is direct and essential in $R_{Z(R)^{*}}$. Then $K \cap M_{n}=(0)$ for all $n \geq h$ since $d_{Z(R)}\left(M_{n}\right)=d_{Z(R)}\left(M_{b}\right)<\infty$. Using the $Z(R)$-embedding $R \rightarrow$ $Z(R)^{(m)}$, we see that $R /\left(K \oplus M_{h}\right)$ is unfaithful over $Z(R)$. This provides a nonzero $d \in Z(R)$ such that $R d \subseteq K \oplus M_{b}$. It is easily seen that $M_{n} \cap$ 
$\left(R d+M_{b}\right)=M_{b}$ for all $n \geq h$. So, $M_{n} / M_{b} \cong\left(R d+M_{n}\right) /\left(R d+M_{b}\right)$ as $T$ modules. Since $R / R d$ is Noetherian over $S$ and so over $T$, it follows that the chain $\left\{M_{n}: n \geq 1\right\}$ stops. Thus $R_{T}$ is Noetherian which makes $T$ a Noetherian ring. By Lemma $2, R_{S}$ is a Noetherian module.

The following theorem improves upon Eisenbud's generalization of of Eakin's theorem.

4. Theorem. Let $S$ be a subring of a ring $R$. Assume that there exists a finite subset $\left\{u_{1}, \ldots, u_{m}\right\}$ of $R$ such that $u_{i} S=S u_{i}$ for $1 \leq i \leq m$ and $R=\Sigma\left\{u_{i} S: 1 \leq i \leq m\right\}$. Then a right $R$-module is Noetherian (resp. has a composition series) if and only if it is Noetherian (resp. has a composition series) as a right $S$-module. Every simple right $R$-module is finite dimensional and semisimple as a right $S$-module.

Proof. After a trivial adjustment if necessary, we may assume that $u_{1}=1$.

Let $M$ be a Noetherian right $R$-module such that every proper $R$-homomorphic image of $M$ is Noetherian over $S$. If possible, let $M$ contain an infinite direct sum $\bigoplus\left\{w_{n} S: n \geq 1\right\}$ of nonzero $S$-submodules. Using Zorn's lemma, we get a $S$-submodule $W$ of $M$ such that the sum $W+$ $\Sigma\left\{w_{n} S: n \geq 1\right\}$ is direct and essential in $M_{S}$. We claim that, for each $k(1 \leq k \leq m)$, we have $d_{k} \in S$ and $n_{k} \in \mathbf{Z}^{+}$such that $w_{1} d_{k} \neq 0$ and $w_{1} d_{k} u_{i} \in W+\Sigma\left\{w_{n} S: 1 \leq n \leq n_{k}\right\}$ for $i=1, \cdots, k$. The claim is trivial for $k=1$ since $u_{1}=1$. Inductively assume that the claim is valid for $k, 1 \leq$ $k<m$. If $w_{1} d_{k} u_{k+1}=0$ then it suffices to take $d_{k}=d_{k+1}$ and $n_{k}=n_{k+1}$. If $w_{1} d_{k} u_{k+1} \neq 0$ then there exists $s \in S$ such that $0 \neq w_{1} d_{k} u_{k+1} s \in W+$ $\Sigma\left\{w_{n} S: n \geq 1\right\}$. Since $u_{i} S=S u_{i}$ for $1 \leq i \leq m$, we have $s^{\prime}, s_{1}, \ldots, s_{k}$, in $S$ such that

$$
s^{\prime} u_{k+1}=u_{k+1} s, \quad s^{\prime} u_{i}=u_{i} s_{i} \text { for } i=1, \ldots, k \text {. }
$$

Set $d_{k+1}=d_{k} s^{\prime}$. Then $0 \neq w_{1} d_{k} u_{k+1} s=w_{1} d_{k+1} u_{k+1} \in W+\Sigma\left\{w_{n} s: n \geq 1\right\}$. Clearly, $w_{1} d_{k+1} \neq 0$. Further, we can choose $n_{k+1} \geq n_{k}$ such that $w_{1} d_{k+1} u_{k+1} \in W+\Sigma\left\{w_{n} S: 1 \leq n \leq n_{k+1}\right\}$. Then, for $1 \leq i \leq k$, we have

$$
w_{1} d_{k+1} u_{i}=w_{1} d_{k} u_{i} s_{i} \in W+\sum\left\{w_{n} s: 1 \leq n \leq n_{k+1}\right\}_{\bullet}
$$

This completes the induction on $k$ and proves our claim. It follows that

$$
\text { (0) } \neq w_{1} d_{m} R \subseteq W+\sum\left\{w_{n} S: 1 \leq n \leq n_{m}\right\} .
$$

Since $M / w_{1} d_{m} R$ is Noetherian over $S$ so also is $M /\left[W+\Sigma\left\{w_{n} S: 1 \leq n \leq n_{m}\right\}\right]$ 
which is impossible since it contains an infinite direct sum of nonzero $S$ submodules. We are thus forced to conclude that $M$ is a finite dimensional $S$-module.

Let $N$ be an essential $S$-submodule of $M$. As in the above paragraph, we obtain a nonzero $R$-submodule of $M$ contained in $N$. Thus $M / N$ is a Noetherian $S$-module. By Lemma $1, M$ is a Noetherian $S$-module.

Now a routine Noetherian induction shows that any Noetherian right $R$-module remains Noetherian over $S$. The converse is trivial.

Let $M$ be a simple right $R$-module. As seen above, $M_{S}$ is Noetherian and so finite dimensional. Let $\left\{L_{n}: n \geq 1\right\}$ be a descending chain of $S$ submodules of $M$. There exists a positive integer $h$ such that $d_{S}\left(L_{n}\right)=$ $d_{S}\left(L_{h}\right)$ for all $n \geq h$. Also, there exists a $S$-submodule $K$ of $M$ such that the sum $K+L_{b}$ is direct and essential in $M_{s}$. Then, for all $n \geq h, K+L_{n}$ is direct and essential in $M_{S}$. Imitating the argument in the second paragraph of the proof and using the simplicity of $M_{R}$, it follows that $M=K \oplus$ $L_{n}$ for all $n \geq h$. So, $L_{n}=L_{b}$ for all $n \geq h$. Thus $M_{S}$ is Artinian as well. So, soc $M_{S}$ is finite dimensional and essential in $M_{S}$. A repetition of the above argument yields $M=\operatorname{soc} M_{S}$. The remaining part is trivial.

The following corollary is a slight generalization of Clifford's theorem [2, p. 343].

5. Corollary. Let $S$ be a subring of a commutative ring $R$ such that $R$ is finitely generated as a $S$-module and let $H$ be a normal subgroup of $i$ nite index in a group $G$. Then any simple right $R(G)$-module is finite dimensional and semisimple as a $S(H)$-module.

\section{REFERENCES}

1. J. E. Björk, On Noetherian and Artinian chain conditions of associative rings, Arch. Math. 24 (1973), 366-378.

2. C. Curtis and I. Reiner, Representation theory of finite groups and associative algebras, Pure and Appl. Math., vol. 11, Interscience, New York, 1962. MR $26 \# 2519$.

3. P. M. Eakin, Jr., The converse to a well-known theorem on Noetherian rings, Math. Ann. 177 (1968), 278-282. MR 37 \#1360.

4. D. Eisenbud, Subrings of Artinian and Noetherian rings, Math. Ann. 185 (1970), 247-249. MR 41 \#6885.

5. E. Formanek, Faithful Noetherian modules, Proc. Amer. Math. Soc. 41 (1973), 381-383.

6. ——; Noetherian P. I. rings, Communications in Algebra 1 (1974), 79-86.

7. A. W. Goldie, The structure of Noetherian rings, Lectures on Rings and Modules, Lecture Notes in Math., vol. 246, Springer-Verlag, New York, 1972. 
8. R. Gordon, Ring theory, Academic Press, New York, 1972.

9. I. Kaplansky, Commutative rings, Allyn and Bacon, Boston, Mass., 1970. MR $40 \# 7234$.

10. M. Nagata, A type of subrings of a noetherian ring, J. Math. Kyoto Univ. 8 (1968), 465-467. MR $38 \# 4460$.

11. C. Procesi and L. W. Small, Endomorphism rings of modules over PIalgebras, Math. Z. 106 (1968), 178-180. MR 38 \#2167.

INSTITUTE FOR ADVANCED STUDY, PRINCETON, NEW JERSEY 08540

DEPARTMENT OF MATHEMATICS, CORNELL UNIVERSITY, ITHACA, NEW YORK 14850

Current address (E. Formanek): Istituto Matematico, Università di Pisa, Pisa, Italy 